ARTICLE

https://doi.org/10.1038/s41467-019-10501-9

\title{
Microscopic ion migration in solid electrolytes revealed by terahertz time-domain spectroscopy
}

Tomohide Morimoto', Masaya Nagai (10 1,2, Yosuke Minowa (1) 1, Masaaki Ashida1,3, Yoichiro Yokotani ${ }^{3}$, Yuji Okuyama ${ }^{4} \&$ Yukimune Kani ${ }^{5}$

Terahertz spectroscopy is one of the most suitable methods for the analysis of electron transport in solids, and has been applied to various materials. Here, we demonstrate that terahertz spectroscopy is the technique of choice to characterize solid electrolytes. We measure the terahertz conductivity of stabilized zirconia, a widely used solid electrolyte material, by terahertz time-domain spectroscopy at high temperatures, providing a wealth of information unavailable from conventional techniques. It is found that the conductivity reflects the microscopic motion of the ion just before hopping to an unoccupied site. Our results suggest a powerful approach in probing the ionic conduction mechanism and could help us explore other solid electrolytes for fuel cells and all-solid-state batteries.

\footnotetext{
${ }^{1}$ Graduate School of Engineering Science, Osaka University, Toyonaka, Osaka 560-8531, Japan. ${ }^{2}$ Panasonic Science Research Alliance Laboratories, Graduate School of Engineering, Osaka University, Suita, Osaka 565-0871, Japan. ${ }^{3}$ Institute for Transdisciplinary Graduate Degree Programs, Osaka University, Toyonaka, Osaka 560-8531, Japan. ${ }^{4}$ Faculty of Engineering, Department of Environmental Robotics, University of Miyazaki, 1-1 Gakuenkibanadai-nishi, Miyazaki 889-2192, Japan. ${ }^{5}$ Technology Innovation Division, Panasonic Corporation, 3-1-1 Yagumo-nakamachi, Moriguchi City, Osaka 570-8501, Japan. Correspondence and requests for materials should be addressed to M.N. (email: mnagai@mp.es.osaka-u.ac.jp)

or to M.A. (email: ashida@mp.es.osaka-u.ac.jp)
} 
T erahertz (THz) conductivity measurements are one of the most powerful and popular tools in present material science to evaluate the carrier transport in solids ${ }^{1-3}$. At low temperatures, the electrons and holes in a doped semiconductor are trapped by the impurity atoms. Here, the resonance frequencies appear in the $\mathrm{THz}$ regime (typically several $\mathrm{THz}$ ). When the carriers are thermally activated at high temperatures, the resulting free electrons and holes exhibit a large conductivity at zero frequency. Since the electronic transport in semiconductors is governed by electron-lattice scattering on the sub-picosecond timescale, the electronic conductivity has also a component in the $\mathrm{THz}$ frequency range ${ }^{4,5}$. The schematics of the electron transport and the corresponding conductivity spectra are shown in Fig. 1a, $\mathrm{b}$, respectively. Recent advances in ultrashort laser and frequency conversion technologies have enabled coherent $\mathrm{THz}$ wave generation and detection ${ }^{2,6,7}$, which allows us to perform THz timedomain spectroscopy (THz-TDS) measurements with high spatio-temporal resolution without need of electrodes. $\mathrm{THz}$ conductivity measurements have been performed on various electronic materials including strong correlated electron systems ${ }^{1,4,8}$.

We propose THz-TDS as an advanced method for the characterization of ion transport in solid electrolytes, which is decisive for the performance of fuel cells or all-solid-state battery ${ }^{9-11}$. Fuel cells have been getting enormous attentions as a new energy generator, because they have various advantages such as high efficiency and cleanliness, compared to conventional power sources $^{12,13}$. All-solid-state battery also has much benefits, such as higher charging speed and higher stability than the conventional batteries ${ }^{14}$. Here, we picked up the stabilized zirconia as an example of solid electrolyte. The stabilized zirconia is the one of the most popular and familiar solid electrolytes and is widely used in commercial solid oxide fuel cells (SOFCs) ${ }^{15,16}$. The ion conduction is usually interpreted in terms of hopping as shown in Fig. 1c. Acceptor dopants located at the cation sublattice in the crystal generate oxygen vacancies, and an adjacent oxygen can
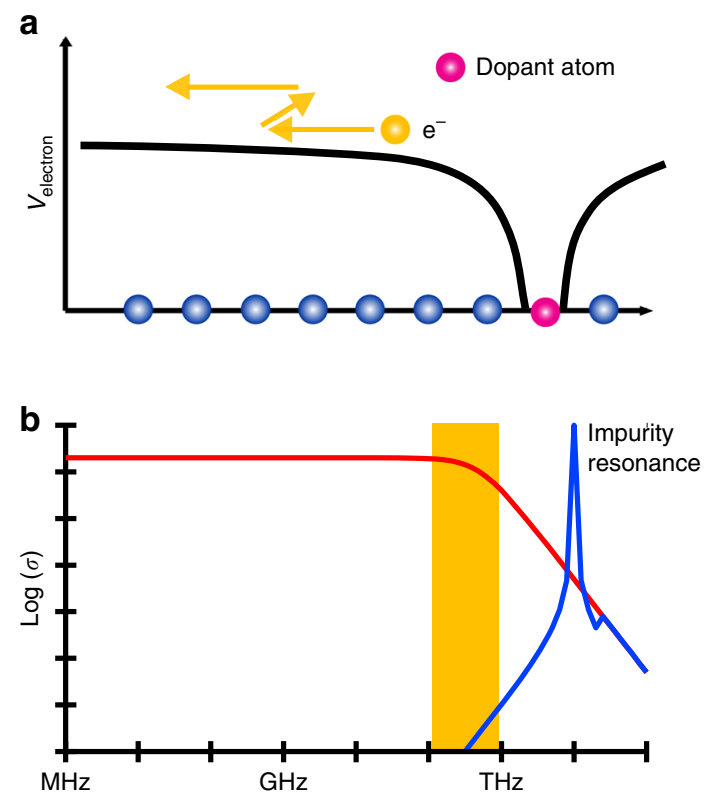

hop to an unoccupied site at adequately high temperatures. The energy of this potential barrier is also termed migration energy, which is one of the most important parameters for the intrinsic ion conduction ${ }^{17}$. However, even at the operating temperature of the SOFCs, some oxygen vacancies form defect clusters and stay at specific sites near the acceptor dopants ${ }^{18}$. The stability of the defect cluster is expressed with the binding energy, which strongly modulates the shape of the long-range potential of the acceptor ion. The long-range potential (also called activation energy $E_{\text {a }}^{\text {low }}$ indicated with the blue dashed line in Fig. 1c) is the sum of migration energy and binding energy, and can be evaluated using conventional low-frequency $(<\mathrm{MHz})$ impedance measurements. The THz-TDS allows us to investigate the microscopic potential in the oxide ion conductor (that is, the potential that is involved in the hopping process indicated with the orange arrow in Fig. 1c), which is crucial for SOFC material design.

We believe the motion of the ions in the $\mathrm{THz}$ frequency range reflects the microscopic ion hopping. Oxygen ions near the vacancy oscillate thermally at the so-called attempt frequency (several THz) before hopping occurs. Such an ion motion can be regarded as a single vacancy mode in accordance with the picture of the hole in an electronic system. It is similar to the localized ion mode in a semiconductor ${ }^{19}$, which is different from the collective ion motion (phonon) ${ }^{1,20-22}$. Since the vacancy modes have strong anharmonicity ${ }^{23}$, a vacancy oscillation with a large amplitude at high temperature induces the hopping to adjacent sites. The frequency components slightly below $1 \mathrm{THz}$ in the conductivity spectra become apparent at high temperatures when the amplitude of the localized vacancy mode is large ${ }^{24-26}$. The expected conductivity spectra at low and high temperatures are shown in Fig. 1d. Therefore, the $\mathrm{THz}$ conductivity in a solid electrolyte contains essential information on the individual ion hopping, which is strongly different from the information provided by the long-range low-frequency conductivity measurements.

Here, in this work, we perform THz-TDS for stabilized zirconia at high temperatures and show that the evaluated conductivity
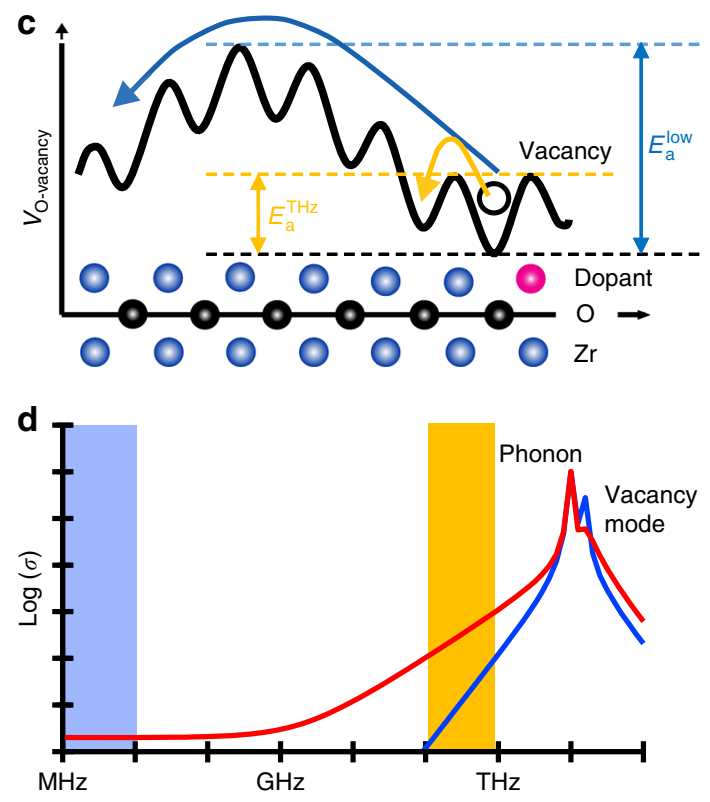

Fig. 1 Schematics of electronic and ionic transport. a Potential for the electron near a donor atom in a semiconductor. $\mathbf{b}$ Corresponding conductivity spectra

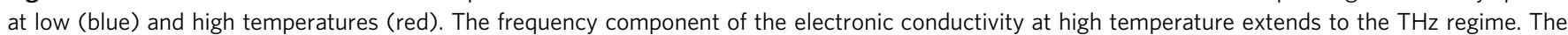
shaded area shows the sub-THz frequency region. c Potential for the oxygen vacancy near a dopant atom in an oxide ion conductor. d Corresponding conductivity spectra at low (blue) and high temperatures (red). The broadening of the vacancy mode towards low frequencies at high temperatures provides information on the individual ion hopping (orange shaded region), which is different from the information obtained in long-range ion transport measurements for low-frequency conductivity (blue shaded region) 
includes the information on the microscopic motion of the ion just before hopping to an unoccupied site occurs, which cannot easily be evaluated by conventional techniques.

\section{Results}

$\mathrm{THz}$ conductivity measurements. We employ THz-TDS to investigate the conductivity in yttria-stabilized zirconia $\mathrm{Zr}_{0.84} \mathrm{Y}_{0.16} \mathrm{O}_{2-\delta}$ (8YSZ), which is commercially used as SOFC electrolyte with the cubic fluorite structure ${ }^{15,27}$ shown in Fig. 2a. Pure $\mathrm{ZrO}_{2}$ undergoes a phase transition from the monoclinic structure to the tetragonal structure at a temperature $T$ of about $1400 \mathrm{~K}$, and transits to the cubic structure at $\sim 2570 \mathrm{~K}^{27,28}$. The doping with $8 \mathrm{~mol} \% \mathrm{Y}_{2} \mathrm{O}_{3}$ enables stabilization of the zirconia's cubic fluorite structure even at room temperature ${ }^{27}$ and simultaneously creates oxygen vacancies. Therefore, the oxygen ions can move to the vacancy site, which constitutes the ion conduction. The ion conduction in 8YSZ has been investigated by conventional impedance measurements ${ }^{29-37}$, and $8 \mathrm{YSZ}$ is actually utilized as solid electrolyte in oximeters and fuel cells ${ }^{15}$. The ion transport in 8 YSZ has also been discussed theoretically ${ }^{17,38}$. Furthermore, the phonon modes have been determined by neutron scattering 39,40 and Fourier infrared spectroscopy ${ }^{41,42}$.

Figure $2 \mathrm{~b}$ shows the time profile of the reference $\mathrm{THz}$ pulse that is detected without the sample, and Fig. $2 \mathrm{c}$ shows the $\mathrm{THz}$ waves that are detected after the reference pulses have passed through the $370 \mu \mathrm{m}$-thick ceramic $8 Y S Z$ plate at different temperatures. The details of our experimental setup for the THz-TDS are described in the Methods. The amplitude of the $\mathrm{THz}$ pulse that is transmitted through the zirconia sample at room temperature (Fig. 2b; blue curve) is smaller than that of the incident (reference) pulse and also exhibits a retardation of $6 \mathrm{ps}$. The amplitude reduction is a result of the reflection and absorption by the sample and the time delay reflects the slow phase velocity of the $\mathrm{THz}$ pulse in $8 \mathrm{YSZ}^{22}$. We find that, as the sample's temperature is increased to $1273 \mathrm{~K}$ the amplitude decreases further. This suggests that the absorption or conductivity in $8 \mathrm{YSZ}$ changes at higher temperatures.

In order to perform a quantitative analysis of the change in the conductive properties, we evaluate the $\mathrm{THz}$ conductivity $\sigma_{\mathrm{THz}}$ from Fourier-transformed electric field profiles of the detected $\mathrm{THz}$ pulses (see Methods). Figure 3a plots the real part of the $\mathrm{THz}$ conductivity in $8 \mathrm{YSZ}$ at different temperatures under ambient atmosphere on a double-logarithmic scale. The real part of the $\mathrm{THz}$ conductivity and the real part of the dielectric constant are shown on a linear scale in Supplementary Fig. 1a and
$1 b$, respectively. At room temperature, the $\mathrm{THz}$ conductivity increases with the frequency $v$. Additionally, also an increase in the temperature leads to an increase of the $\mathrm{THz}$ conductivity, in particular, at the lower frequency region and hence the slopes of the spectra decrease at higher temperatures. Using a single crystal, we verified that these trends hardly depend on the crystallinity (see Supplementary Fig. 2; the THz conductivity of an 8YSZ single crystal at various temperatures exhibits the same spectral shapes as that of the ceramic sample discussed here).

The origin of the $\mathrm{THz}$ conductivity component at high temperatures is important for the physics of the SOFC electrolyte. One may consider that this conductivity component is a result of the spectral broadening of the high-frequency phonon mode. Actually, one infrared-active transverse optical (TO) phonon mode at $7.7 \mathrm{THz}$ has been theoretically predicted for the cubic zirconia crystal ${ }^{28}$. Its spectral width should be proportional to the temperature via inter-phonon scattering ${ }^{22,37}$ while maintaining $\sigma \propto \nu^{2}$ at the frequency region below the resonance frequency. The $\mathrm{THz}$ conductivity spectrum at room temperature is indeed proportional to $v^{2}$ (Fig. 2a). However, the slope of the spectrum decreases with temperature, and approaches $\sigma \propto \nu$ at high temperatures. This suggests that the spectral change is not due to the spectral broadening of the phonon.

To confirm the minor contribution of the phonon to the $\mathrm{THz}$ conductivity at high temperatures, we measure the $\mathrm{THz}$ conductivity of another zirconia sample with a $\mathrm{Y}_{2} \mathrm{O}_{3}$ doping concentration of $3 \mathrm{~mol} \%$ (hereafter referred to as $3 \mathrm{YSZ}$ ). It is known that the $3 \mathrm{YSZ}$ has an additional phonon resonance at 5 $\mathrm{THz}$ due to the tetragonal structure and exhibits a weak ion conduction compared to $8 \mathrm{YSZ}^{15,43}$. Figure $3 \mathrm{~b}$ plots the real part of the $\mathrm{THz}$ conductivity in $3 \mathrm{YSZ}$ at various temperatures on a double-logarithmic scale. The slope of $\sigma$ in the 3YSZ sample remained proportional to $v^{2}$ even at $T=1273 \mathrm{~K}$. This also supports that the spectral change of the $\mathrm{THz}$ conductivity in $8 Y S Z$ is not governed by the phonon resonance.

Evaluation of the activation energy. Another plausible mechanism for the conductivity increase observed in Fig. 3a is the long-range ion conduction that expands toward the $\mathrm{THz}$ frequency region. Ion conduction in SOFC electrolytes is explained with the hopping of some oxygen ions to unoccupied sites in the lattice. Various long-range conduction models, such as the correlated barrier hopping model, have been proposed for the frequency region below $\mathrm{GHz}$. These models predict a power law for the high-frequency conductivity that is similar to our results
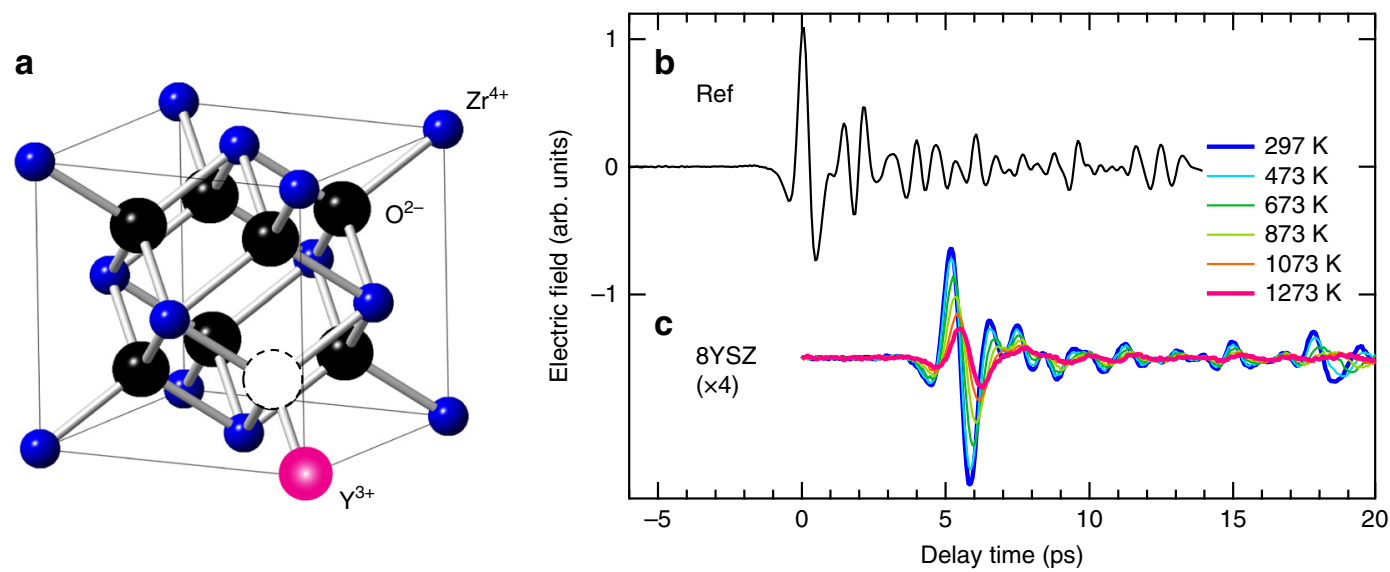

Fig. 2 THz time-domain spectroscopy for an 8YSZ pellet. a Crystal structure of 8YSZ. b The time profile of the reference THz pulse that is obtained without sample. c The time profiles of the THz pulses that have passed through the $370 \mu \mathrm{m}$-thick 8 YSZ pellet at different temperatures. This data set is offset for clarity 

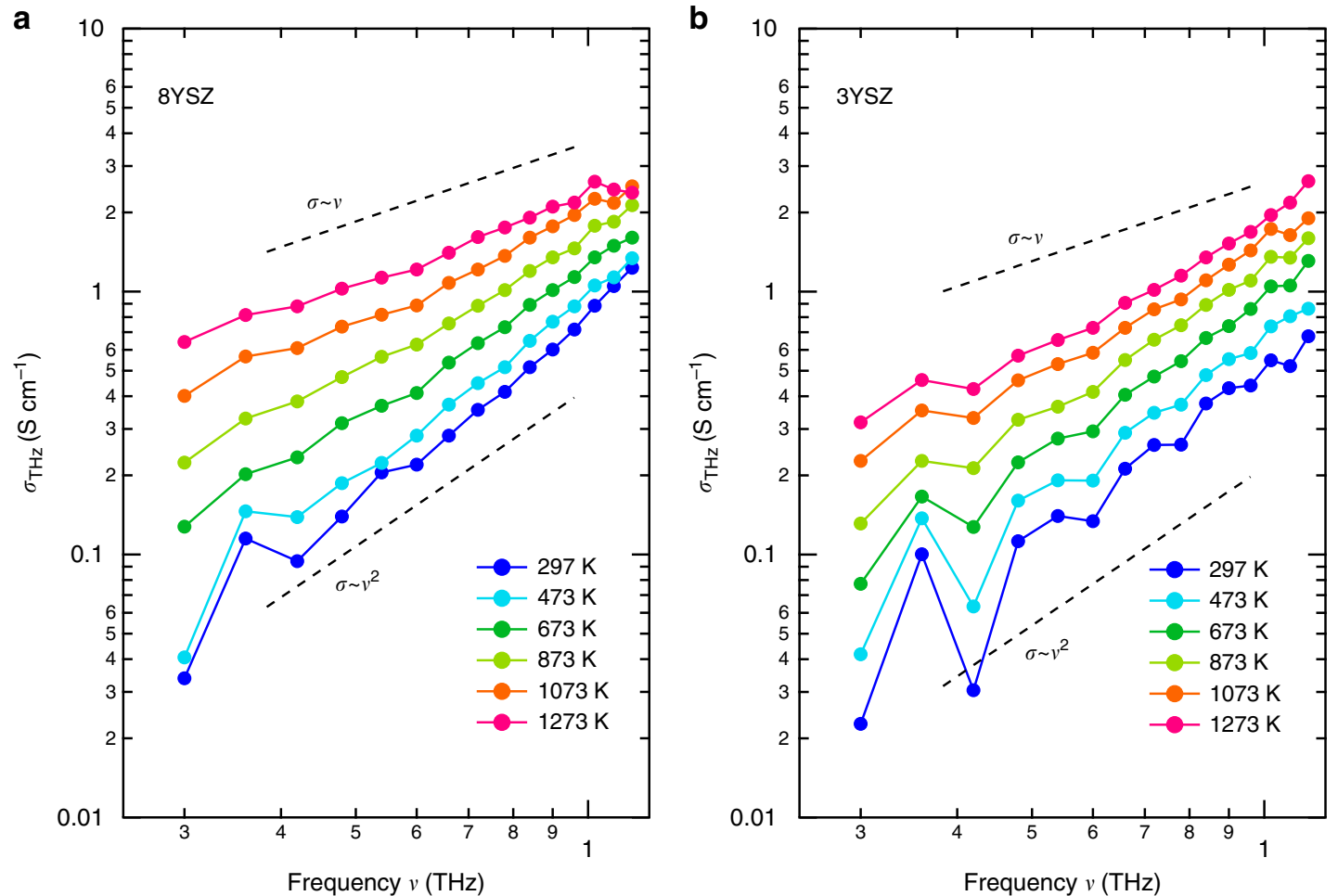

Fig. $3 \mathrm{THz}$ conductivities of 8 YSZ and 3YSZ. a The real parts of the optical conductivities of $8 Y S Z$ and $\mathbf{b}$ those of $3 Y S Z$ at different temperatures. The dashed lines represent the linear and quadratic dependences for reference

$\left(\sigma=\sigma_{0}+\sigma^{s}\right)^{35}$. Similar discussions have been performed for glass systems ${ }^{44}$. The low-frequency ion conductivity $\sigma$ can be analyzed with the Arrhenius model using

$$
\sigma=(A / T) \exp \left[-E_{a} / k_{B} T\right]
$$

where $A, k_{B}$, and $E_{a}$ correspond to the pre-exponential factor, the Boltzmann coefficient, and the activation energy, respectively. The red closed circles in Fig. 4a present the THz conductivity at $0.36 \mathrm{THz}$. Here, the horizontal axis expresses the inverse temperature and the vertical axis plots $\ln (\sigma T)$. Since Eq. (1) can be rewritten as $\ln (\sigma T)=-\left(E_{a} / k_{B}\right)(1 / T)+\ln A$, a straight line in this plot suggests that the conductivity obeys the Arrhenius model at high temperatures. In the low-temperature regime, the measured $\mathrm{THz}$ conductivity does not follow the straight line due to the phonon contribution as mentioned above. However, as elaborated in the next Section, for the present purpose it is sufficient to fit the high-temperature range. From the slope at high temperatures, we can estimate the activation energy at $v=0.36 \mathrm{THz}$, $E_{a}^{T H z}(0.36 \mathrm{THz})=0.3 \mathrm{eV}$. Furthermore, Fig. $4 \mathrm{~b}$ evidences that the activation energy decreases as the $\mathrm{THz}$ frequency increases. We also show those for the 8YSZ single crystal and 3YSZ sample in Supplementary Fig. 3, and the prominent features are the same.

Low-frequency impedance measurements. This activation energy evaluated from the $\mathrm{THz}$ conductivity is different from the results obtained by conventional low-frequency impedance measurements. We performed impedance measurements on an 8YSZ ceramic sample and show the Arrhenius plot of the lowfrequency conductivity as the blue circles in Fig. 4a. Obviously, the slope of the low-frequency conductivity is larger than that of the THz conductivity. We evaluated an activation energy of $E_{a}^{\text {low }}$ $=1.03 \mathrm{eV}$, which is consistent with the $0.8-1.1 \mathrm{eV}$ reported previously ${ }^{31,33-35}$. Therefore, the origin of the activation energy obtained by the $\mathrm{THz}$ conductivity measurement is different from that obtained by the low-frequency impedance measurements.

\section{Discussion}

As elaborated further below, a vacancy oscillates at a specific site of the lattice until it jumps to an adjacent site. The latter process occurs at a timescale of nanoseconds or longer. This hopping mechanism suggests that the $\mathrm{THz}$ conductivity component is related to the large-amplitude vacancy modes, which are relevant for the vacancy hopping. These vacancies modulate phonons. While one TO phonon in cubic zirconia without dopant has been predicted at $7.7 \mathrm{THz}^{28}$, infrared reflection spectroscopy implies a TO phonon mode at 10 to $12 \mathrm{THz}$ in $\mathrm{YSZ}^{41,42}$. We also experimentally confirmed this resonance (at $11 \mathrm{THz}$, as shown in Supplementary Fig. 4). On the other hand, Shin and Ishigame et al. have revealed defect-induced modes around 9 and $21 \mathrm{THz}$ by employing hyper Raman scattering spectroscopy ${ }^{45}$. These modes are infrared-active $T_{1 u}$ modes, and have been assigned to the oxygen defect with the analysis of the $\mathrm{O}_{6}$ molecule ${ }^{45}$. Thus, we analyze the infrared-active component with the assumption of two contributions, the phonon component with low-nonlinearity and the oxygen vacancy component. Since the potentials for the vacancy modes exhibit a strong anharmonicity ${ }^{17,38}$, the vacancy jumps to the adjacent sites via large-amplitude modes after many attempts. Hence the conductivity spectrum of the vacancy mode extends to low frequencies, and the activation energy at $v=0 \mathrm{~Hz}$ extrapolated from the $\mathrm{THz}$ conductivity includes the information of the microscopic ion hopping.

Let us verify the $\mathrm{THz}$ conductivity originating from this vacancy motion by using the conventional analytical technique of the $\mathrm{THz}$ vibrational modes in an anharmonic potential 25,26 . While the actual shape of the potential is very complex ${ }^{17,38}$, we approximate the individual potential at each site as Morse potential, which is one of the most typical anharmonic potentials ${ }^{46}$. The energy level $E_{i}$ of the $i$-th vibrational mode in the Morse potential can be described as $E_{i}=(i+1 / 2) h \nu_{0}(1-\chi(i+$ $1 / 2)$ ) in the lowest-order approximation, leading to the following transition energy between the $i$-th level and $i+1$-th 
a

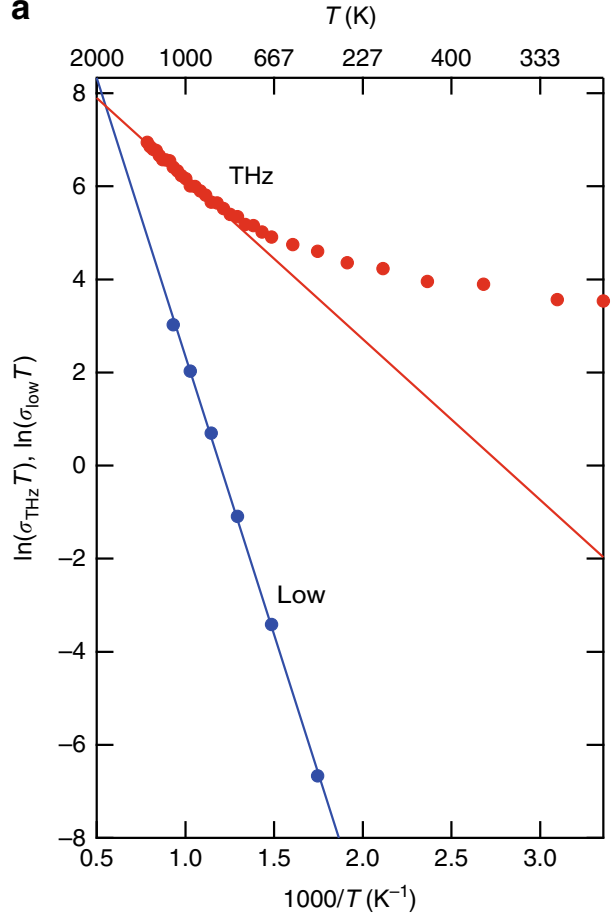

b

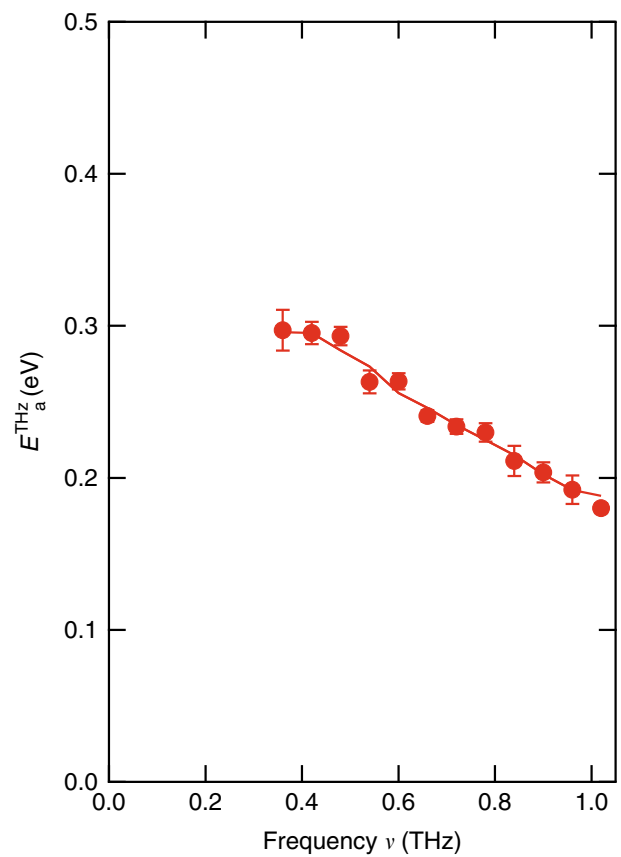

Fig. 4 Temperature dependence of the THz conductivity in 8YSZ. a Red closed circles represent the temperature dependence of the THz conductivity in $8 Y S Z$ at $0.36 \mathrm{THz}$. Blue closed circles show the conventionally measured low-frequency conductivity in 8YSZ. The solid lines are the fitting results obtained using the Arrhenius equation. $\mathbf{b}$ The data points show the activation energy evaluated from the THz conductivity of 8YSZ at different frequencies. The solid smoothing curve is a guide to the eye

level: $E_{i, i+1}=h \nu_{i, i+1}=h \nu_{0}(1-2 \chi(i+1))$. Here, $v_{0}$ represents the frequency of the lowest vibrational level (i.e., the attempt frequency), and $\chi$ is the anharmonic coefficient ${ }^{46}$. In this model, the depth of the potential $U$ is $h \nu_{0} / 4 \chi$, and the quantum number is limited by $i<1 / 2 \chi-1$, which can be derived from the condition $\nu_{\mathrm{i}, \mathrm{i}+1}>0$. By assuming that the population of the vibrational mode, $N_{i, i+1}$, obeys the Maxwell-Boltzmann distribution, the optical conductivity spectrum (which is directly proportional to the absorption) at the frequency $v$ can be written as follows

$$
\begin{gathered}
\sigma \propto \nu \sum_{i=0}\left\{\mu_{i, i+1}^{2}\left(N_{i}-N_{i+1}\right) \gamma\left(\nu-\nu_{i, i+1}\right)\right\} \\
\propto \frac{\nu \sum_{i=0}\left\{(i+1)\left(e^{-\frac{E_{i}}{k_{B} T}}-e^{-\frac{E_{i+1}}{k_{B} T}}\right) \gamma\left(\nu-\nu_{i, i+1}\right)\right\}}{\sum_{i=0} e^{-\frac{E_{i}}{k_{B} T}}},
\end{gathered}
$$

where $\mu_{i, i+1} \propto \sqrt{i+1}$ represents the transition dipole moment and $\gamma\left(\nu-\nu_{i, i+1}\right)$ the line width of the $i$-th transition. If we assume that the conductivity for $\nu<1 \mathrm{THz}$ is mainly governed by a single transition between a certain $i$-th level and the $i+1$-th level, Eq. (2) can be simplified as

$$
\sigma \propto \nu_{i+1, i}\left(N_{i+1}-N_{i}\right) \approx\left(\frac{1}{h k_{B} T}\right) e^{-\frac{E_{i}}{k_{B} T}}
$$

in the low-frequency limit of $\left(E_{i+1}-E_{i}\right) / k_{B} T \ll 1$. This relation is equivalent to equation (1), which was used to determine the $\mathrm{THz}$ activation energy from the Arrhenius plot (Fig. 4b). Therefore, the activation energy $E_{a}^{T H z}(0.36 \mathrm{THz})=0.3 \mathrm{eV}$ corresponds to the energy $E_{i}$. Since the sum of zero-point vibration energy $\left(h \nu_{0} / 2=0.02 \mathrm{eV}\right.$ with the assumption of $\left.\nu_{0}=11 \mathrm{THz}\right)$ and $E_{i}$ in the low-frequency limit is close to the potential barrier $U$ for ion hopping, we find $U \approx 0.32 \mathrm{eV}$.

To clarify whether the above assumptions are valid for this material or not, we simulate the spectra of the $\mathrm{THz}$ conductivity using the model outlined above. We set the potential depth to $U=$ $0.32 \mathrm{eV}$, use $v_{0}=11 \mathrm{THz}$ as experimentally estimated, and assume $\chi=0.035$ using the relationship $U=h \nu_{0} / 4 \chi$. Figure 5 a shows the energy diagram of the Morse potential, and Fig. $5 \mathrm{~b}$ shows the mode populations at 300 and $1200 \mathrm{~K}$ considering the Maxwell-Boltzmann distribution. We can derive the $\mathrm{THz}$ conductivity from the mode population using Eq. (2), and the results are shown in Fig. $5 \mathrm{c}$. In the $\mathrm{THz}$ regime, the components of the conductivity of the localized vacancy mode increase with the temperature, and the conductivity is proportional to the frequency below $1 \mathrm{THz}$. Figure $5 \mathrm{~d}$ is the Arrhenius plot of the calculated $\mathrm{THz}$ conductivity at $0.36 \mathrm{THz}$. Indeed, this figure shows a linear relation with an activation energy close to the experimental value $E_{0}^{T H z}(0.36 \mathrm{THz})=0.27 \mathrm{eV}$. We also simulated the spectra of the $\mathrm{THz}$ conductivity using different parameters while maintaining the potential depth. The evaluated activation energy is slightly smaller than the depth of the potential due to the temperature-dependent normalization factor $\sum \exp \left[-E_{i} / k_{B} T\right]$ in Eq. (2). In spite of the simple potential shape, the numerical simulation reproduces the experimental results.

Next, let us specify the site whose potential barrier is reflected in the $\mathrm{THz}$ conductivity measurements. When the vacancy moves to a neighboring site, it passes the saddle point of the potential that lies between two cations ${ }^{30}$. An oxygen vacancy at high temperatures remains at the lowest energy site for a long time because even the thermal energy of the vacancy mode at $1000 \mathrm{~K}$ is significantly smaller than the energy scale of the binding energy $\left(>0.3 \mathrm{eV}^{18,47}\right)$. This fact suggests that the activation energy $E_{a}^{T H z}$ evaluated from the $\mathrm{THz}$ conductivity reflects the minimum potential barrier for the oxygen vacancy at the lowest energy site (indicated with the orange dashed line in Fig. 1c). We note that the expected potential barrier near the dopants is larger than the intrinsic migration energy due to the additional binding energy $^{23,48}$. However, it has been reported that the lowest energy site of the oxygen vacancy is the second-nearest neighbor of the 

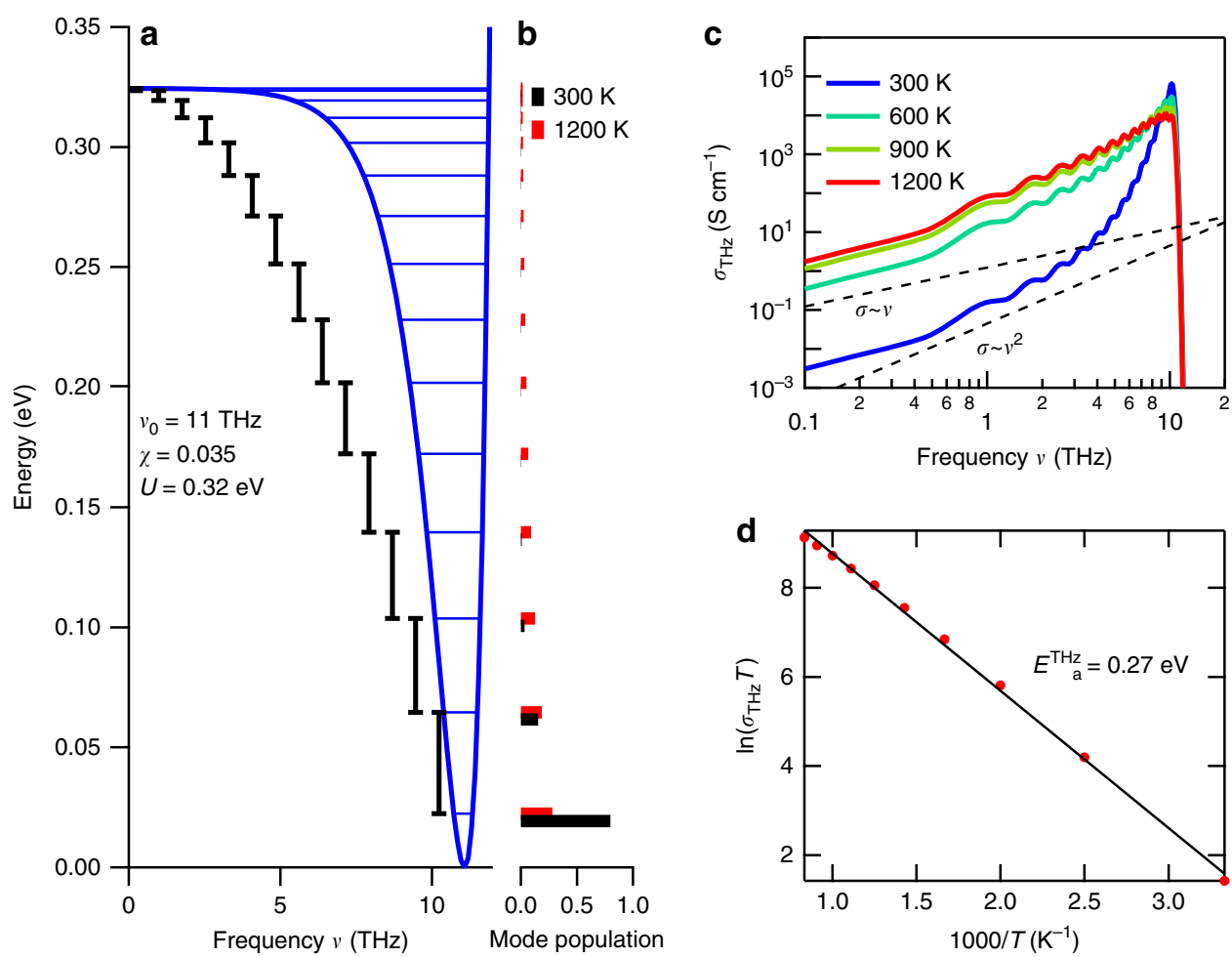

Fig. $5 \mathrm{THz}$ ion motions in the Morse potential. a The simplified potential shape and energy levels for the localized vacancy mode with $\nu_{0}=11 \mathrm{THz}$ and $\chi=0.035$. $\mathbf{b}$ The populations of the energy levels are shown in black and red for 300 and $1200 \mathrm{~K}$, respectively. c The calculated conductivity spectra for the localized vacancy mode. d The Arrhenius plot for the calculated $\mathrm{THz}$ conductivity at $0.36 \mathrm{THz}$

dopant in case of an impurity with a large ionic radius ${ }^{18,47}$. In this case, there are several equivalent sites (located like a shell around the impurity) with the same minimum energy and the vacancy can move to a neighboring site with the same energy via several paths. The potential barrier at the $\mathrm{Zr}-\mathrm{Zr}$ saddle point ${ }^{17}$ is the minimum energy required for the hopping to the next equivalent site. Therefore, we consider that activation energy determined by the $\mathrm{THz}$ conductivity measurements reflects the intrinsic migration energy of the oxygen vacancy.

The abovementioned migration and binding energies, which are relevant for microscopic ion conduction, have been investigated extensively, but the exact values are still a matter of debate in theoretical studies ${ }^{30,48}$. Both parameters have been characterized experimentally using the temperature-dependent slopes of the Arrhenius plots for the low-frequency conductivity ${ }^{49,50}$. However, the evaluated values are likely to exhibit a large uncertainty due to a simplified analysis, leading to unresolved issues even in zirconia ${ }^{16}$. The mechanisms of oxide ion migration (e.g. the formation of oxygen vacancies and their preferential locations) have also been investigated by nuclear magnetic resonance ${ }^{51}$, and muon measurements ${ }^{52}$. However, these are not standard techniques that are easily accessible for a wide range of researchers. Here, we applied a standard technique based on ultrashort optical pulse technology and directly evaluated the oxide ion dynamics in the solid electrolyte of SOFC. This method enables the direct evaluation of the charge fluctuation owning to its high temporal resolution. Our proposed method is very attractive for the field of ionics, because the intrinsic migration energy of the oxygen vacancy can be accurately measured. We measured the $\mathrm{THz}$ conductivity for different stabilized zirconia samples to investigate the dependence of the activation energy on the impurity atom type $\left(M_{2} \mathrm{O}_{3}\right.$ with $M=\mathrm{Y}, \mathrm{Yb}, \mathrm{Gd}, \mathrm{Sc}$, and $M_{2}$ with $M=\mathrm{Ca}, \mathrm{Mg}$ ). Figure 6a shows the activation energies evaluated by the low-frequency impedance method, $E_{a}^{l o w}$. The temperature dependence of the low-frequency impedance is shown in Supplementary Fig. 5. Figure $6 \mathrm{~b}$ shows the activation energies evaluated by the THz conductivity measurements at $\nu=0.36 \mathrm{THz}$, $E_{a}^{T H z}$. The graph clearly shows that the $\mathrm{THz}$ activation energy decreases with the ionic radius of the dopant, which is different from the result obtained in the impedance measurements. This is because the activation energy obtained in the impedance measurement reflects the long-range potential while the $\mathrm{THz}$ activation energy reflects the short-range potential. The trend of the $\mathrm{THz}$ activation energy is a result of the increase in the effective lattice constant due to the larger ionic radius of the dopant. Therefore, the migration energy along the $\mathrm{Zr}-\mathrm{Zr}$ path decreases ${ }^{17,48}$. This tendency is in good agreement with the calculation result ${ }^{17}$.

In conclusion, we measured the $\mathrm{THz}$ conductivity of various stabilized zirconia samples by $\mathrm{THz}$-TDS. We observed the $\mathrm{THz}$ responses originating from the large-amplitude oxygen-vacancy motion, which is related to the vacancy hopping to adjacent sites. The temperature dependence of the $\mathrm{THz}$ conductivity revealed the intrinsic migration energy of the vacancy. This method is applicable to various solid electrolytes in protonic ceramic fuel cells (PCFC) $)^{53-57}$ and all-solid-state lithium ion batteries ${ }^{10,58}$, because the attempt frequencies of the ion transport in these materials are also expected to lie around several $\mathrm{THz}^{59}$. Since THz-TDS should reveal the hidden mechanism of ionic conduction, our proposed method is useful for development of novel solid electrolytes for fuel cells or all-solid-state batteries.

\section{Methods}

Sample preparation. The $\mathrm{Zr}_{0.84} \mathrm{Y}_{0.16} \mathrm{O}_{2-\delta}$ and $\mathrm{Zr}_{0.88} \mathrm{Sc}_{0.12} \mathrm{O}_{2-\delta}$ pellets were prepared using commercial powder. The $\mathrm{Zr}_{0.84} \mathrm{Y}_{0.16} \mathrm{O}_{2-\delta}$ powder $(\mathrm{TOSOH})$ and the $\mathrm{Zr}_{0.88} \mathrm{Sc}_{0.12} \mathrm{O}_{2-\delta}$ powder (DAIICHI KIGENSO KAGAKU KOGYO) were first pressed into pellets at $250 \mathrm{MPa}$ and then sintered at $1673 \mathrm{~K}$ for 5 and $2 \mathrm{~h}$ in air, respectively. The $\mathrm{Zr}_{0.84} M_{0.16} \mathrm{O}_{2-\delta}(M=\mathrm{Mg}, \mathrm{Yb}, \mathrm{Ca})$ samples were prepared by a solid-state reaction method (SSR). The reagent-grade $\mathrm{ZrO}_{2}$ (99.9\%), $\mathrm{MgO}(99.9 \%)$, $\mathrm{Yb}_{2} \mathrm{O}_{3}(99.9 \%)$, and $\mathrm{CaCO}_{3}(99.9 \%)$ powders were weighed and mixed in ethanol using a mortar and a pestle. The powder mixtures were calcined in air for $5 \mathrm{~h}$ at $1273 \mathrm{~K}$. The calcined $\mathrm{Zr}_{0.84} M_{0.16} \mathrm{O}_{2-\delta}(M=\mathrm{Mg}, \mathrm{Yb}, \mathrm{Ca})$ powders were ball-milled 


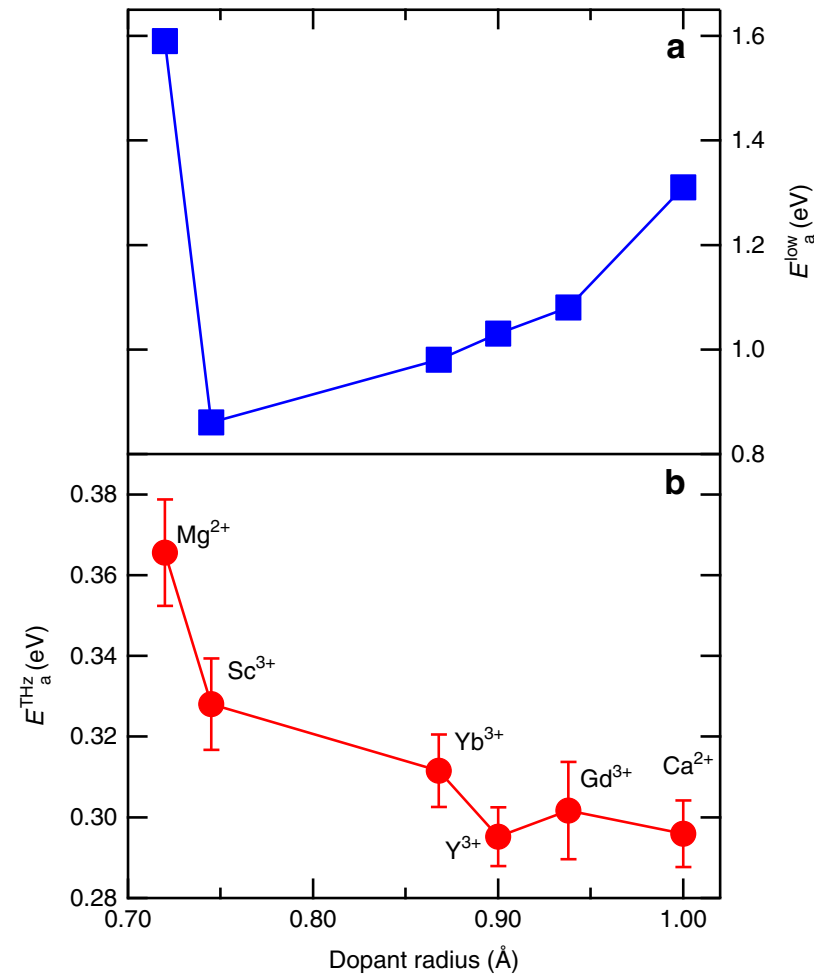

Fig. 6 The activation energies for stabilized zirconia samples with different dopant ions. a The low-frequency activation energy obtained by impedance measurements. $\mathbf{b}$ The activation energy in the $\mathrm{THz}$ regime obtained by THz-TDS

and pressed into pellets at $250 \mathrm{MPa}$ and then sintered at $1873 \mathrm{~K}$ for $5 \mathrm{~h}$ in air. The $\mathrm{Zr}_{0.84} \mathrm{Gd}_{0.16} \mathrm{O}_{2-\delta}$ pellets with single phase were prepared by a combustion synthesis method. $\mathrm{ZrO}\left(\mathrm{NO}_{3}\right)_{3} 2.1 \mathrm{H}_{2} \mathrm{O}(99.9 \%), \mathrm{Gd}\left(\mathrm{NO}_{3}\right)_{3} 6.1 \mathrm{H}_{2} \mathrm{O}(99.9 \%)$, water, citric acid, and ethylenediamine tetraacetice acid were mixed while maintaining a $\mathrm{pH}$ of 10 by adding aqueous ammonia. After ammonium nitrate was added, the solution was heated at $623 \mathrm{~K}$. The obtained powders were calcined in air for $10 \mathrm{~h}$ at $1173 \mathrm{~K}$. The powder was further ball-milled and pressed into CIP pellets at $250 \mathrm{MPa}$, which was followed by sintering at $1873 \mathrm{~K}$ for $10 \mathrm{~h}$ in air. The obtained samples were used for both the THz conductivity measurements and the impedance measurements. For characterization, we measured the X-ray diffraction (XRD) of the powder samples using MinFlex600 (Rigaku) with CuKa radiation. All XRD patterns shown in Supplementary Fig. 6 were analyzed by employing the Rietveld refinement technique. The X-ray powder diffraction analysis confirmed a well-defined fluorite pattern for all samples prepared in this study.

Experimental setup for the THz time-domain spectroscopy. We performed the THz-TDS measurements by using the output from an Er-doped fiber laser (TOPTICA Photonics, FemtoFiber pro NIR) with a center wavelength of $780 \mathrm{~nm}$ (second harmonic of $1560 \mathrm{~nm}$ ), a repetition rate of $80 \mathrm{MHz}$, an average power of $130 \mathrm{~mW}$, and a pulse duration of $90 \mathrm{fs}$. A beam splitter was used to divide the output beam into the excitation beam for $\mathrm{THz}$ pulse generation and the sampling beam for detection, respectively. The excitation pulse was focused on an InAs plate exposed to a magnetic field of $0.5 \mathrm{~T}$. The $\mathrm{THz}$ pulses emitted from InAs were guided by four parabolic mirrors to the sample and transmitted to an electro-optic (EO) detector, which was a $1-\mathrm{mm}$-thick (110)-oriented $\mathrm{ZnTe}$ crystal. The instantaneous $\mathrm{THz}$ electric field amplitude alters the birefringence of the $\mathrm{ZnTe}$ crystal The sampling pulses were focused on the same crystal, and the polarization state of the sampling pulse modulated by the $\mathrm{THz}$ wave was measured using a quarter wave plate, a Wolston prism, and balanced Si detectors. To achieve a high signal-to-noise ratio in the measurements, the excitation pulses were chopped at $6 \mathrm{kHz}$ and the modulated signal was analyzed with a lock-in amplifier. The sample was placed in an electric furnace with temperatures up to $1300 \mathrm{~K}$ under atmosphere without drygas replacement, and the THz pulse entered the furnace through an aperture with 7 $\mathrm{mm}$ diameter (no window) using an off-axis parabolic mirror. This setup has a low-frequency detection limit of $0.35 \mathrm{THz}$. The black-body radiation from the sample can hardly influence our measurements because its field amplitude is much lower than that of the coherent THz pulse. Nevertheless, we carefully blocked the black-body radiation from the electric furnace with thin black polypropylene films.
Evaluation of complex optical conductivity. Using THz-TDS, we determined the electric field waveform of the THz pulse transmitted through the sample, $E_{s}(t)$, and that of the reference pulse without sample, $E_{r}(t)$. The complex optical conductivity $\tilde{\sigma}(\omega)$ can directly be evaluated with these time profiles as explained in the following. First, we only employ the measured electric field waveforms in the time region from 2 to $16 \mathrm{ps}$, because the signal at $18 \mathrm{ps}$ caused by multiple reflections in the sample complicates the analysis. Second, the complex transmittance $\tilde{t}(\omega)$, which is a ratio between the Fourier-transformed electric fields $\tilde{E}_{s}(\omega)$ and $\tilde{E}_{r}(\omega)$, can simply be expressed in terms of the complex permittivity $\tilde{\varepsilon}(\omega)$,

$$
\tilde{t}(\omega)=\frac{\tilde{E}_{s}(\omega)}{\tilde{E}_{r}(\omega)}=\frac{4 \sqrt{\tilde{\varepsilon}(\omega)}}{(\sqrt{\tilde{\varepsilon}(\omega)}+1)^{2}} \exp \left[\frac{i(\sqrt{\tilde{\varepsilon}(\omega)}-1) d \omega}{c}\right]
$$

Here, $c$ is the speed of light (in vacuum) and $d$ is the sample thickness. Thus, we can obtain $\tilde{\varepsilon}(\omega)$ from $\tilde{t}(\omega)$ and then evaluate the complex conductivity $\tilde{\sigma}(\omega)$ using the following relation,

$$
\tilde{\varepsilon}(\omega)=\varepsilon_{\infty}+\frac{\tilde{\sigma}(\omega)}{i \omega}
$$

Impedance analysis. The electrical conductivities of the samples were measured by a two-probe alternating-current technique. The samples prepared for this measurement had the shape of a pellet (length: $0.5-1.0 \mathrm{~mm}$ ). The Pt electrodes were prepared by applying a Pt paste on both surfaces of the pellet and subsequent baking at $1173 \mathrm{~K}$ in air. The samples were equilibrated with $1.9 \% \mathrm{H}_{2} \mathrm{O}-21 \% \mathrm{O}_{2}-\mathrm{Ar}$ in the temperature range of $573-1073 \mathrm{~K}$. The complex impedance was measured in the frequency range of $4-8 \mathrm{MHz}$ using an inductance-capacitance-resistance meter (HIOKI corporation: IM3536)

\section{Data availability}

All data needed to evaluate the conclusions in the paper are present in the paper and/or its Supplementary Information. All other data are available from the corresponding author upon reasonable request.

Received: 9 October 2018 Accepted: 15 May 2019

Published online: 17 June 2019

\section{References}

1. Ferguson, B. \& Zhang, X. C. Materials for terahertz science and technology. Nat. Mater. 1, 26-33 (2002).

2. Tonouchi, M. Cutting-edge terahertz technology. Nat. Photonics 1, 97-105 (2007).

3. Dhillon, S. S. et al. The2017 terahertz science and technology roadmap. J. Phys D. Appl. Phys. 50, 043001 (2017).

4. Grischkowsky, D., Keiding, S., van Exter, M. \& Fattinger, C. Far-infrared timedomain spectroscopy with terahertz beams of dielectrics and semiconductors. J. Opt. Soc. Am. B 7, 2006-2015 (1990).

5. Lloyd-Hughes, J. \& Jeon, T. I. A review of the terahertz conductivity of bulk and nano-materials. J. Infrared, Millim., Terahertz Waves 33, 871-925 (2012).

6. Sakai, K. Terahertz Optoelectronics. (Springer, Berlin, 2005).

7. Mittleman, D. M. Frontiers in terahertz sources and plasmonics. Nat. Photonics 7, 666-669 (2013).

8. Huber, R. et al. How many-particle interactions develop after ultrafast excitation of an electron-hole plasma. Nature 414, 286-289 (2001).

9. Steele, B. C. H. \& Heinzel, A. Materials for fuel-cell technologies. Nature 414, 345-352 (2001).

10. Kamaya, N. et al. A lithium superionic conductor. Nat. Mater. 10, 682-686 (2011).

11. Knauth, P. Inorganic solid Li ion conductors: an overview. Solid State Ion. 180, 911-916 (2009).

12. Mahato, N., Banerjee, A., Gupta, A., Omar, S. \& Balani, K. Progress in material selection for solid oxide fuel cell technology: a review. Prog. Mater. Sci. 72, 141-337 (2015).

13. Minh, N. Q. Ceramic fuel cells. J. Am. Ceram. Soc. 76, 563-588 (1993).

14. Sun, C., Liu, J., Gong, Y., Wilkinson, D. P. \& Zhang, J. Recent advances in allsolid-state rechargeable lithium batteries. Nano Energy 33, 363-386 (2017).

15. Weber, A. \& Ivers-Tiffée, E. Materials and concepts for solid oxide fuel cells (SOFCs) in stationary and mobile applications. J. Power Sources 127, 273-283 (2004).

16. Kharton, V. V., Marques, F. M. B. \& Atkinson, A. Transport properties of solid oxide electrolyte ceramics: a brief review. Solid State Ion. 174, 135-149 (2004).

17. Pornprasertsuk, R., Ramanarayanan, P., Musgrave, C. B. \& Prinz, F. B. Predicting ionic conductivity of solid oxide fuel cell electrolyte from first principles. J. Appl. Phys. 98, 103513 (2005). 
18. Zacate, M. O., Minervini, L., Bradfield, D. J., Grimes, R. W. \& Sickafus, K. E. Defect cluster formation in $\mathrm{M}_{2} \mathrm{O}_{3}$-doped cubic $\mathrm{ZrO}_{2}$. Solid State Ion. 128, 243-254 (2000).

19. Klingshirn, C. Semiconductor Optics. (Springer, Berlin, 2005).

20. Stoyanov, N. S., Ward, D. W., Feurer, T. \& Nelson, K. A. Terahertz polariton propagation in patterned materials. Nat. Mater. 1, 95-98 (2002).

21. Feurer, T., Vaughan, J. C. \& Nelson, K. A. Spatiotemporal coherent control of lattice vibrational waves. Science 299, 374-377 (2003).

22. Ichikawa, Y., Nagai, M. \& Tanaka, K. Direct observation of the soft-mode dispersion in the incipient ferroelectric $\mathrm{KTaO}_{3}$. Phys. Rev. B-Condens. Matter Mater. Phys. 71, 092106 (2005).

23. Eichler, A. Tetragonal Y-doped zirconia: structure and ion conductivity. Phys. Rev. B-Condens. Matter Mater. Phys. 64, 174103 (2001).

24. Walther, M., Fischer, B. M. \& Jepsen, P. U. Noncovalent intermolecular forces in polycrystalline and amorphous saccharides in the far infrared. Chem. Phys. 288, 261-268 (2003).

25. Korter, T. M. \& Plusquellic, D. F. Continuous-wave terahertz spectroscopy of biotin: vibrational anharmonicity in the far-infrared. Chem. Phys. Lett. 385, 45-51 (2004).

26. Jewariya, M., Nagai, M. \& Tanaka, K. Ladder climbing on the anharmonic intermolecular potential in an amino acid microcrystal via an intense monocycle terahertz pulse. Phys. Rev. Lett. 105, 203003 (2010).

27. Liu, T., Zhang, X., Wang, X., Yu, J. \& Li, L. A review of zirconia-based solid electrolytes. Ionics 22, 2249-2262 (2016).

28. Zhao, X. \& Vanderbilt, D. Phonons and lattice dielectric properties of zirconia. Phys. Rev. B-Condens. Matter Mater. Phys. 65, 075105 (2002).

29. Abelard, P. \& Baumard, J. F. Study of the dc and ac electrical properties of an yttria-stabilized zirconia single crystal $\left[\left(\mathrm{ZrO}_{2}\right)_{0.88^{-}}\left(\mathrm{Y}_{2} \mathrm{O}_{3}\right)_{0.12}\right]$. Phys. Rev. B 26, 1005-1017 (1982).

30. Kilner, J. A. \& Brook, R. J. A study of oxygen ion conductivity in doped nonstoichiometric oxides. Solid State Ion. 6, 237-252 (1982).

31. Badwal, S. P. S. Electrical conductivity of single crystal and polycrystalline yttria-stabilized zirconia. J. Mater. Sci. 19, 1767-1776 (1984).

32. Stafford, R. J., Rothman, S. J. \& Routbort, J. L. Effect of dopant size on the ionic conductivity of cubic stabilised $\mathrm{ZrO}_{2}$. Solid State Ion. 37, 67-72 (1989).

33. Ciacchi, F. T., Crane, K. M. \& Badwal, S. P. S. Evaluation of commercial zirconia powders for solid oxide fuel cells. Solid State Ion. 73, 49-61 (1994).

34. Filal, M., Petot, C., Mokchah, M., Chateau, C. \& Carpentier, J. L. Ionic conductivity of yttrium-doped zirconia and the 'composite effect'. Solid State Ion. 80, 27-35 (1995).

35. Pimenov, A., Ullrich, J., Lunkenheimer, P., Loidl, A. \& Ruscher, C. H. Ionic conductivity and relaxations in $\mathrm{ZrO}_{2}-\mathrm{Y}_{2} \mathrm{O}_{3}$ solid solutions. Solid State Ion. 109, 111-118 (1998).

36. Yamamoto, O. et al. Zirconia based oxide ion conductors for solid oxide fuel cells. Ionics 4, 403-408 (1998).

37. Teranishi, T. et al. Complex conductivity using wideband spectroscopy for yttria/ ytterbia- stabilized zirconia ceramics. Jpn. J. Appl. Phys. 51, 011102 (2012).

38. Shimojo, F., Okabe, T., Tachibana, F., Kobayashi, M. \& Okazaki, H. Molecular dynamics studies of yttria stabilized zirconia: I. Structure and oxygen diffusion. J. Phys. Soc. Jpn. 61, 2848-2857 (1992).

39. Elcombet, M. A neutron scattering investigation of Cubic Stabilised Zirconia (CSZ) - II. Lattice dynamics of Y- and Ca-CSZ. J. Phys. Chem. Solids 57, 343-351 (1996).

40. Cousland, G. P. et al. Investigation of the vibrational properties of cubic yttriastabilized zirconia: a combined experimental and theoretical study. J. Phys. Chem. Solids 75, 351-357 (2014)

41. Liu, D. W., Perry, C. H. \& Ingel, R. P. Infrared spectra in nonstoichiometric yttria-stabilized zirconia mixed crystals at elevated temperatures. J. Appl. Phys. 64, 1413-1417 (1988).

42. Artemov, V. G. et al. Analysis of electric properties of $\mathrm{ZrO}_{2}-\mathrm{Y}_{2} \mathrm{O}_{3}$ single crystals using teraherz IR and impedance spectroscopy techniques. Russ. J. Electrochem. 50, 690-693 (2014).

43. Feinberg, A. \& Perry, C. H. Structural disorder and phase transitions in $\mathrm{ZrO}_{2}$ $\mathrm{Y}_{2} \mathrm{O}_{3}$ system. J. Phys. Chem. Solids 42, 513-518 (1981).

44. Krishnaswami, S., Jain, H., Kamitsos, E. I. \& Kapoutsis, J. A. Connection between the microwave and far infrared conductivity of oxide glasses. J. Non Cryst. Solids 274, 307-312 (2000).

45. Shin, S. \& Ishigame, M. Defect-induced hyper-Raman spectra in cubic zirconia. Phys. Rev. B 34, 8875-8882 (1986).

46. Haken, H. \& Wolf, H. C. Molecular Physics and Elements of Quantum Chemistry. (Springer, Berlin, 1995).

47. Khan, M. S., Islam, M. S. \& Bates, D. R. Cation doping and oxygen diffusion in zirconia: a combined atomistic simulation and molecular dynamics study. $J$. Mater. Chem. 8, 2299-2307 (1998).

48. Nakayama, Masanobu \& Martion., Manfred First-principles study on defect chemistry and migration of oxide ions in ceria doped with rare-earth cations. Phys. Chem. Chem. Phys. 11, 3241 (2009).
49. Bauerle, J. E. \& Hrizo, J. Interpretation of the resistivity temperature dependence of high purity $\left(\mathrm{ZrO}_{2}\right)_{0.90}\left(\mathrm{Y}_{2} \mathrm{O}_{3}\right)_{0.10}$. J. Phys. Chem. Solids 30, 565-570 (1969).

50. Omar, S., Wachsman, E. D., Jones, J. L. \& Nino, J. C. Crystal structure-ionic conductivity relationships in doped ceria systems. J. Am. Ceram. Soc. 92, 2674-2681 (2009).

51. Kawata, K., Maekawa, H., Nemoto, T. \& Yamamura, T. Local structure analysis of YSZ by Y-89 MAS-NMR. Solid State Ion. 177, 1687-1690 (2006).

52. Vieira, R. B. L. et al. Muon-Spin-rotation study of yttria-stabilized zirconia $\left(\mathrm{ZrO}_{2}: \mathrm{Y}\right)$ : evidence for muon and electron separate traps. J. Phys. Conf. Ser. 551, 012050 (2014).

53. Duan, C. et al. Readily processed protonic ceramic fuel cells with high performance at low temperatures. Science 349, 1321-1326 (2015).

54. Kreuer, K. D. Proton-conducting oxides. Annu. Rev. Mater. Res. 33, 333-359 (2003).

55. Bae, K. et al. Demonstrating the potential of yttrium-doped barium zirconate electrolyte for high-performance fuel cells. Nat. Commun. 8, 14553 (2017).

56. An, H. et al. A $5 \times 5 \mathrm{~cm}^{2}$ protonic ceramic fuel cell with a power density of 1.3 $\mathrm{W} \mathrm{cm}{ }^{-2}$ at $600^{\circ} \mathrm{C}$. Nat. Energy 3, 870-875 (2018).

57. Choi, S. et al. Exceptional power density and stability at intermediate temperatures in protonic ceramic fuel cells. Nat. Energy 3, 202-210 (2018).

58. Kawasoko, H., Shiraki, S., Suzuki, T., Shimizu, R. \& Hitosugi, T. Extremely low resistance of $\mathrm{Li}_{3} \mathrm{PO}_{4}$ electrolyte/ $\mathrm{Li}\left(\mathrm{Ni}_{0.5} \mathrm{Mn}_{1.5}\right) \mathrm{O}_{4}$ electrode interfaces. ACS Appl. Mater. Interfaces 10, 27498-27502 (2018).

59. Bachman, J. C. et al. Inorganic solid-state electrolytes for lithium batteries: mechanisms and properties governing ion conduction. Chem. Rev. 116, 140-162 (2016).

\section{Acknowledgements}

This work was partially supported by JSPS KAKENHI (Grant Numbers JP17J04949, JP15H03579, JP18H05212) and the Ministry of Education, Culture, Sports, Science and Technology under Program for Leading Graduate Schools "Interactive Materials Science Cadet Program".

\section{Author contributions}

T.M. performed the experiments, data analysis, and wrote the initial draft. M.N. codirected the scientific research, provided advice for the $\mathrm{THz}$ spectroscopy and data analysis, and wrote the manuscript. T.M. and M.N. developed the theoretical model with assistance from Y.M. M.A provided advice for the THz spectroscopy and the project direction. Y.Y. developed the electric furnace for the THz spectroscopy. Y.O. prepared some oxide ion conductors and performed X-ray diffraction and impedance measurements. Y.K. conceived the idea of utilizing THz spectroscopy for characterization of solid electrolytes, co-directed the scientific research, organized the joint research project, and prepared some oxide ion conductors. All authors contributed to the discussions for this manuscript and wrote parts of the manuscript.

\section{Additional information}

Supplementary Information accompanies this paper at https://doi.org/10.1038/s41467019-10501-9.

Competing interests: The authors declare no competing interests.

Reprints and permission information is available online at http://npg.nature.com/ reprintsandpermissions/

Peer review information: Nature Communications thanks the anonymous reviewers for their contribution to the peer review of this work.

Publisher's note: Springer Nature remains neutral with regard to jurisdictional claims in published maps and institutional affiliations.

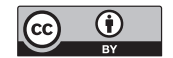

Open Access This article is licensed under a Creative Commons Attribution 4.0 International License, which permits use, sharing, adaptation, distribution and reproduction in any medium or format, as long as you give appropriate credit to the original author(s) and the source, provide a link to the Creative Commons license, and indicate if changes were made. The images or other third party material in this article are included in the article's Creative Commons license, unless indicated otherwise in a credit line to the material. If material is not included in the article's Creative Commons license and your intended use is not permitted by statutory regulation or exceeds the permitted use, you will need to obtain permission directly from the copyright holder. To view a copy of this license, visit http://creativecommons.org/ licenses/by/4.0/.

(C) The Author(s) 2019 